\title{
FEASIBILITY OF USING DIFFERENT IONIC LIQUIDS FOR THE ENZYMATIC SYNTHESIS OF AMOXICILLIN
}

\author{
S. C. PEREIRA ${ }^{1}$, W. KOPP ${ }^{2}$, M. A. I. DURO ${ }^{3}$, S. M. SILVA ${ }^{3}$, R. BUSSAMARA ${ }^{4}$, G. MARIN ${ }^{4}$, \\ J. DUPONT ${ }^{4}$, R. L. C. GIORDANO ${ }^{2}$ and R. C. GIORDANO ${ }^{2}$ \\ ${ }^{1}$ Brazilian Agricultural Research Corporation, Embrapa Instrumentation \\ ${ }^{2}$ Federal University of São Carlos, Chemical Engineering Department \\ ${ }^{3}$ Federal University of Bahia, Department of Chemical Engineering \\ ${ }^{4}$ Federal University of Rio Grande do Sul, Institute of Chemistry \\ E-mail: sandracerqueirapereira@gmail.com
}

\begin{abstract}
Industrially, amoxicillin has been produced by chemical processes. But, the enzymatic synthesis catalyzed by penicillin G acylase (PGA, E.C.3.5.1.11) is more attractive from the environmental point of view. However, an obstacle for the industrial application of the enzymatic route is the limited product yield due to undesirable hydrolytic reactions. This drawback can be minimized by reducing water activity in the reaction medium. Ionic liquids (ILs) have emerged as an alternative to conventional organic media. This study assessed the feasibility of use of different ILs, protic (PILs) and aprotic (AILs), in the enzymatic synthesis of amoxicillin, by testing the stability of reaction substrates (6-aminopenicillanic acid and $p$ hydroxyphenylglycine methyl ester) in these solvents. These compounds were completely stable in AILs. Besides, PGA was active in AILs. In contrast, none of the evaluated PILs was inert for these substrates making unfeasible the use for this enzymatic process.
\end{abstract}

\section{INTRODUCTION}

The current industrial process used to produce the semi-synthetic $\beta$-lactam antibiotics is a rather drastic chemical route, including the protection and de-protection of reactive groups. It demands low temperatures, the use of toxic organochloride solvents, and generates non-recyclable waste (Ospina et al., 1996). The yields that are achieved by the chemical synthesis are very high contributing for the low market prices of these drugs. This is an important feature for public health, especially in non-developed countries. But, the tendency towards stricter environmental regulation opens a window of opportunity for other technologies less harmful. Enzymatic reactions are "environmental-friendly" strategies.

Working under mild conditions of reaction and generating harmless salts as waste of the purification process, the enzymatic synthesis of semi-synthetic $\beta$-lactam antibiotics became a target of intense research as a "cleaner" process. However, it is still not economically competitive with the fine-tuned conventional chemical process (Giordano et al., 2006). In order to reduce costs of the enzymatic route is essential increasing the yield and selectivity (synthesis/hydrolysis ratio). In the kinetically controlled synthesis (KCS) of $\beta$-lactam antibiotics, an activated derivative of the acyl donor (an ester or amide) reacts with the $\beta$-lactam nucleus (6-aminopenicillanic acid, 6-APA) and produces the drug. In this reaction, penicillin $\mathrm{G}$ acylase (PGA) acts as a transferase. 
Nevertheless, PGA is also a hydrolase. As result, selectivity becomes the main drawback for the industrial operation. Figure 1 exemplifies the reactions involved in the KCS of amoxicillin when the $p$-hydroxyphenylglycine methyl ester (PHPGME) is used. These undesirable side reactions could be partially avoided by reducing the water activity $\left(\mathrm{a}_{\mathrm{w}}\right)$ in the reaction medium. One way to reduce the hydrolytic reactions is the use of organic cosolvents. Although some good results have been reported, organic solvents have the disadvantage of being volatile, flammable, toxic, and harmful to the environment. Thus, ionic liquids (ILs) have emerged as solvents that may replace the traditional organic media.

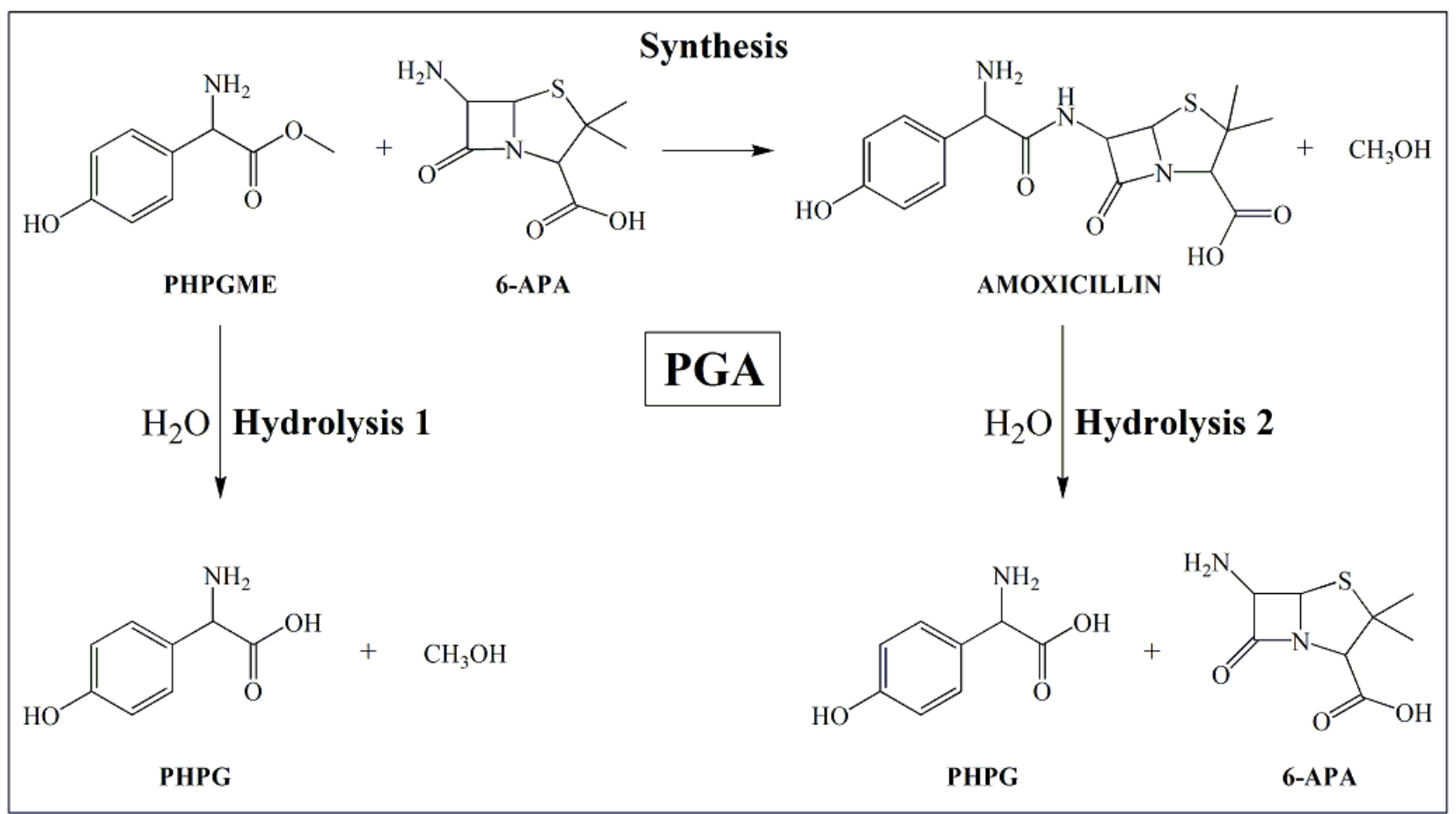

Figure 1 - KCS of amoxicillin catalyzed by penicillin G acylase (PGA).

ILs are organic salts that exist as liquids below a threshold temperature, usually around 100 ${ }^{\circ} \mathrm{C}$ (Quijano et al., 2010). ILs have been considered a promising class of "green solvents" mainly due to their negligible vapor pressure. Moreover, they are non-flammable and have high thermal and chemical stability (Zhang et al., 2011). In addition, ILs have been recognized as "designer solvents", since their physicochemical properties such as hydrophobicity, viscosity, density, and solubility can be tuned by selecting different combinations of cations and anions, and of the attached substituent as well (Zhao, 2010). Typical ILs are based on organic cations paired with a variety of anions that have a strongly delocalized negative charge (Filice et al., 2010).

The imidazolium (IM) based salts are the most investigated ILs in biotechnology. Moreover, bis(trifluoromethylsulfonyl)imide $\left(\mathrm{NTf}_{2}\right)$, tetrafluoroborate $\left(\mathrm{BF}_{4}\right)$, and hexafluorophosphate $\left(\mathrm{PF}_{6}\right)$ are by far the anions most often used (Roosen et al., 2008). These combinations are named of aprotic ionic liquids (AILs). Nevertheless, there is another important category of ILs, named protic 
ionic liquids (PILs), which are produced through the simple combination of a Brønsted acid and a Brønsted base (Olivier-Bourbigou et al., 2010). The main difference between them is the presence of donor and acceptor sites of protons, which are able to build a hydrogen bonds network (Greaves \& Drummond, 2008).

In short, enzymatic synthesis of $\beta$-lactam antibiotics has not gained industrial feasibility yet due to the low selectivity of the reaction, caused by hydrolysis of substrate and product. Consequently, important antibiotics (amoxicillin among them) continue to be produced by the chemical route, using organochloride solvents. Thus, using the "greenest solvent", water, is not an alternative in practice. In this context, the present study assessed the feasibility of use of different ILs, protic (PILs) and aprotic (AILs), in the enzymatic synthesis of amoxicillin, by testing the stability of reaction substrates (6-aminopenicillanic acid and $p$-hydroxyphenylglycine methyl ester) in these solvents.

\section{MATERIALS AND METHODS}

\subsection{Materials}

The chemicals, 6-aminopenicillanic acid (6-APA), p-hydroxyphenylglycine methyl ester (PHPGME), and 6-nitro-3-phenylacetamide benzoic acid (NIPAB) were obtained from SigmaAldrich Corporation (Wisconsin, U.S.). Penicillin G acylase (PGA) from Escherichia coli covalently immobilized on Sepabeads ${ }^{\circledR}$ (Mitsubishi Chemical Corporation) was kindly donated by the Department of Enzymatic Biocatalysis, Institute of Catalysis, CSIC, Spain (Mateo et al., 2007). The aprotic ionic liquids (AILs) were kindly donated by the Laboratory of Molecular Catalysis (Department of Organic Chemistry, Federal University of Rio Grande do Sul). The protic ionic liquids (PILs) were kindly donated by the Laboratory of Applied Thermodynamic (Department of Chemical Engineering, Federal University of Bahia).

\subsection{Methods}

Substrates stability: It was performed the measurement of $\mathrm{a}_{\mathrm{w}}$ of the several ILs that would be assessed in order to guarantee the same operating conditions. The measurement of $a_{w}$ was fixed as 0.8 . Thus, it was necessary to make a correction of the value of $a_{w}$ for some of LIs that were tested, as it will be described later. Having completed this step, each of the different ILs (5 mL) was transferred to individual flasks containing a mass of one of the substrates (6-APA or PHPGME), making a total concentration of $50 \mathrm{mM}$. All these flasks were homogenized and taken for a shaker at $25{ }^{\circ} \mathrm{C}$ and $350 \mathrm{rpm}$ for 24 hours. The stability of the substrates was monitored continuously through the areas of the chromatographic bands (see analytical method).

Thermodynamic water activity $\left(\mathrm{a}_{\mathrm{w}}\right)$ : A volume of each solvent was added to a cell reading accommodated in a dew point sensor, which had been previously equilibrated at $25^{\circ} \mathrm{C}$. Since the measurement of $\mathrm{a}_{\mathrm{w}}$ was less than 0.8 , it became necessary to add water in order to reach the desired reading. After each addition of water, the system was homogenized and equilibrated for 24 hours before performing a new reading. The hygrometer was continuously calibrated with the aid of standard solutions (Decagon Devices Incorporated).

Enzymatic stability: A mass of $500 \mathrm{mg}$ of the biocatalyst (PGA immobilized on Sepabeads ${ }^{\circledR}$ ) was incubated in $5 \mathrm{~mL}$ of each reaction medium, all presenting $\mathrm{a}_{\mathrm{w}}$ equal to 0.8 (as described 
above). All the flasks were homogenized and taken for a shaker at $25{ }^{\circ} \mathrm{C}$ and $350 \mathrm{rpm}$ for 24 hours. Periodically, aliquots were withdrawn and centrifuged at $12.000 \mathrm{~g}$ and $4{ }^{\circ} \mathrm{C}$ for 5 minutes. A sample of $10 \mathrm{mg}$ of the biocatalyst was used for the dosage of residual activity determined by the method of NIPAB (as described below).

Enzymatic activity: Enzymatic activity of PGA immobilized on Sepabeads ${ }^{\circledR}$ was measured by the 6-nitro-3-phenylacetamide benzoic acid (NIPAB) method at a wavelength of $405 \mathrm{~nm}$ (Kutzbach \& Rauenbusch, 1974). For this purpose, an Ultrospec 2000 UV-Vis spectrophotometer from Pharmacia Biotech was used. The operational conditions were as follows: solution of NIPAB $66 \mathrm{mg} / \mathrm{L}$ in $50 \mathrm{mM}$ phosphate buffer, $\mathrm{pH} 7.5$, and $25^{\circ} \mathrm{C}$. One unit of enzyme $\left(\mathrm{U}_{\mathrm{NIPAB}}\right)$ was defined as the amount of enzyme that hydrolyzes $1 \mu \mathrm{mol}$ de NIPAB per minute at $25^{\circ} \mathrm{C}$ and $\mathrm{pH} 7.5$.

Synthesis of amoxicillin: Enzymatic reactions for the synthesis of amoxicillin were conducted under kinetic control. In these experiments, the reaction between PHPGEE and 6-APA was catalyzed by PGA immobilized on Sepabeads ${ }^{\circledR}$. Initial substrate concentrations were $50 \mathrm{mM}$ for both PHPGEE and 6-APA. In addition, the ratio of enzyme to substrate (E/S) was $52 \mathrm{IU} / \mathrm{mmol}$ of substrate and the total reaction volume was $30 \mathrm{~mL}$; thus, the amount of enzymatic derivative used in all the experiments was $0.3 \mathrm{~g}$. Every synthesis was conducted at $25{ }^{\circ} \mathrm{C}$. Throughout the course of the reactions, aliquots were withdrawn for HPLC analyzes.

Analytical method: Concentrations were determined by HPLC in a Waters 996 Photodiode Array Detector (PDA) liquid chromatograph equipped with a Phenomenex Gemini C18 column $(150 \times 4.6 \mathrm{~mm}, 5 \mu \mathrm{m})$. The mobile phase was prepared as follows: $1.4 \mathrm{~g}$ of sodium dodecyl sulfate (SDS), $0.6805 \mathrm{~g}$ of phosphate monobasic anhydrous $\left(\mathrm{KH}_{2} \mathrm{PO}_{4}\right), 650 \mathrm{~mL}$ of ultrahigh-purity water (Millipore Milli-Q System), $350 \mathrm{~mL}$ of acetonitrile, and correction to $\mathrm{pH} 3.0$ using phosphoric acid $\left(\mathrm{H}_{3} \mathrm{PO}_{4}\right)$. Analyzes were carried out at $25{ }^{\circ} \mathrm{C}$ and detection at $225 \mathrm{~nm}$.

\section{RESULTS AND DISCUSSION}

In order to evaluate a feasibility of using ionic liquids (ILs) in the enzymatic synthesis of amoxicillin, an important antibiotic, it was performed a screening with different types of ILs, including protic (PILs) and aprotic (AILs) in respect to stability of reaction substrates (6-APA and PHPGME). In addition, the stability of the biocatalyst (PGA immobilized on Sepabeads ${ }^{\circledR}$ ) was assessed in the same experimental conditions for those ILs of interest after the previous study of stability of the substrates. It is worth mentioning that 21 PILs and 3 AILs were investigated in this study. Figures 2 and 4 present the chemical structures of the ions that are part of the constitution of the solvents that were evaluated.

An important criterion to aid in selecting a suitable solvent for a given application, which here would be the enzymatic synthesis of amoxicillin, is the stability of the substrates involved in these reactions. In other words, the substrates cannot be degraded in the presence of the solvent to be used as the reaction medium. The results show that in the presence of all AILs, both 6-APA as PHPGME were completely stable. Therefore, it was performed the enzymatic stability study in these AILs. PGA shows to be totally stable and active in AILs. This information demonstrates the feasibility of using these AILs in order to conduct the enzymatic production of amoxicillin, as can be seen in Figure 3, which presents the results to the different conditions of $a_{w}$. In the best condition, an increase of $400 \%$ in selectivity was observed for the reactions carried out with 
BMI.PF 6 (see Figure 2) as cosolvent at $75 \%\left(\mathrm{v}_{\mathrm{IL}} / \mathrm{v}_{\text {water }}\right)$ when compared to the totally aqueous medium (using phosphate buffer).

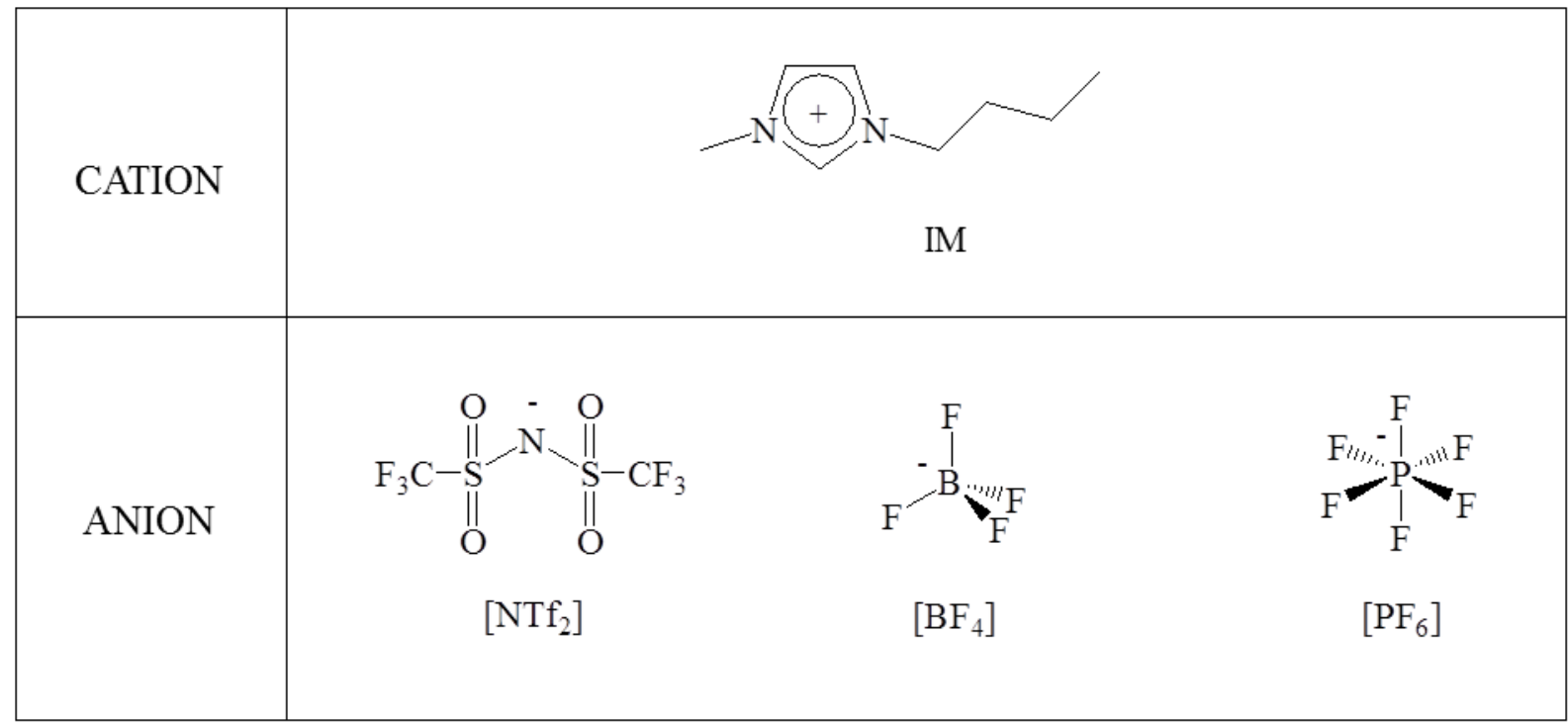

Figure 2 - Chemical structure of the aprotic ionic liquids (AILs).

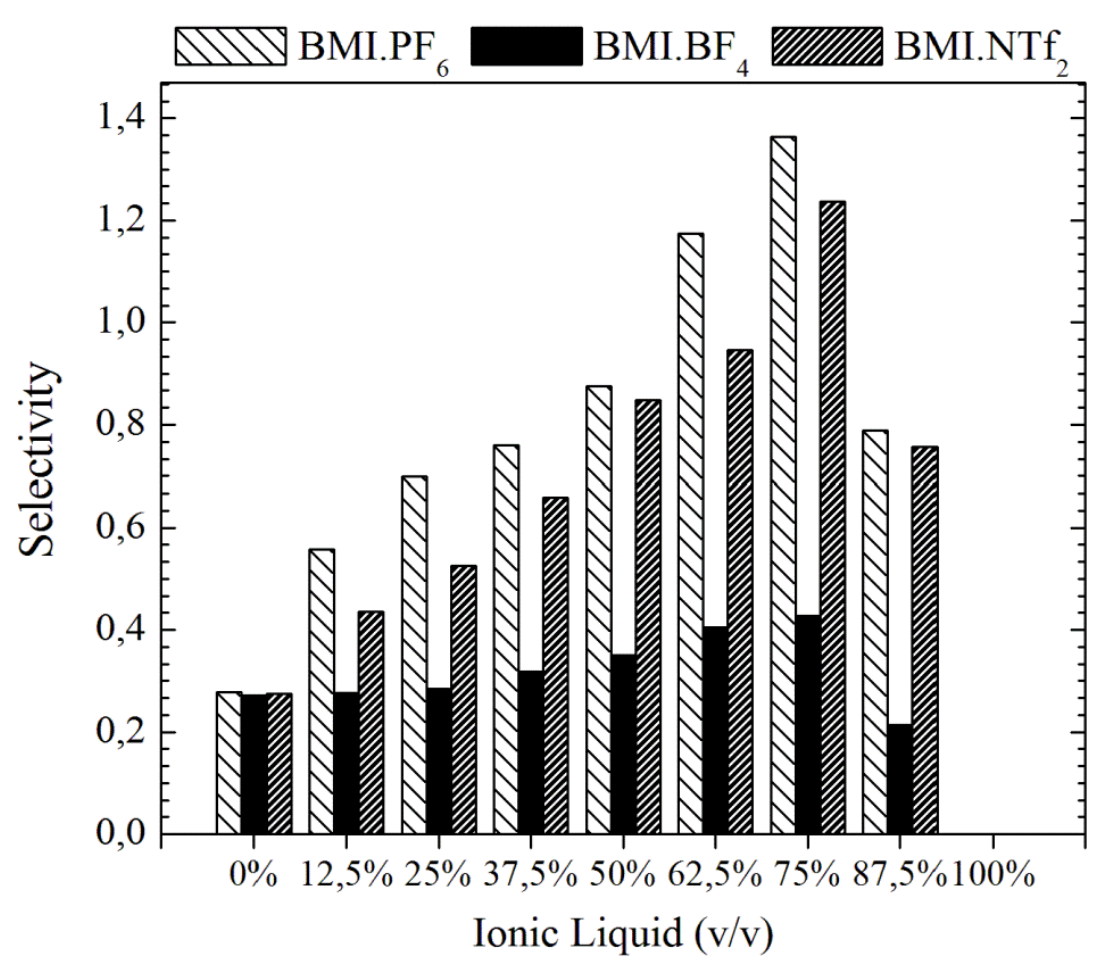

Figure 3 - Selectivity after 180 minutes of amoxicillin enzymatic syntheses. 


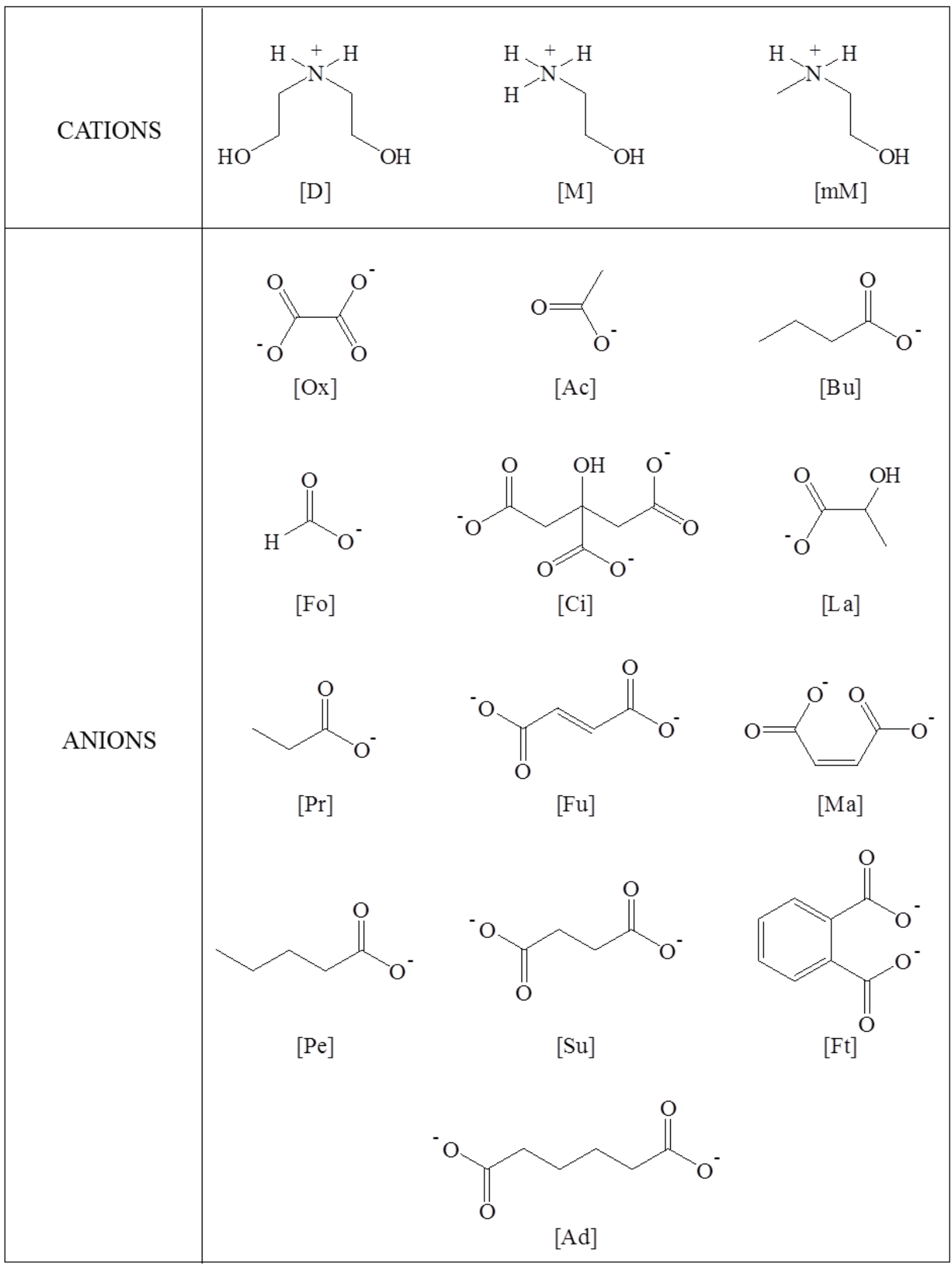

Figure 4 - Chemical structure of the cations and anions that compose the protic ionic liquids (PILs), making a total of 21 solvents assessed in this study for this category. 
In contrast, in the presence of all PILs, both 6-APA as PHPGME were not stable, that is, the substrates of the reaction interact with the solvents. PILs were not inert to the reaction substrates, as one would expect for a reaction medium and thus could not be directly applied to the reaction of enzymatic synthesis of amoxicillin. Because it is a large number of solvents, here we chose to present only one solvent (M.La, see Figure 4) representing the category of PILs and a substrate (PHPGME). However, it is worth mentioning that the profile was similar for all 21 PILs studied. Thus, Figure 5 shows the continuous degradation profile of PHPGME in the presence of M.La, observed by the reduction of the chromatographic band relating to this substrate, and including the formation of another unknown compound at retention time equal to 3.4 minutes, whose identity was not characterized in this present study.

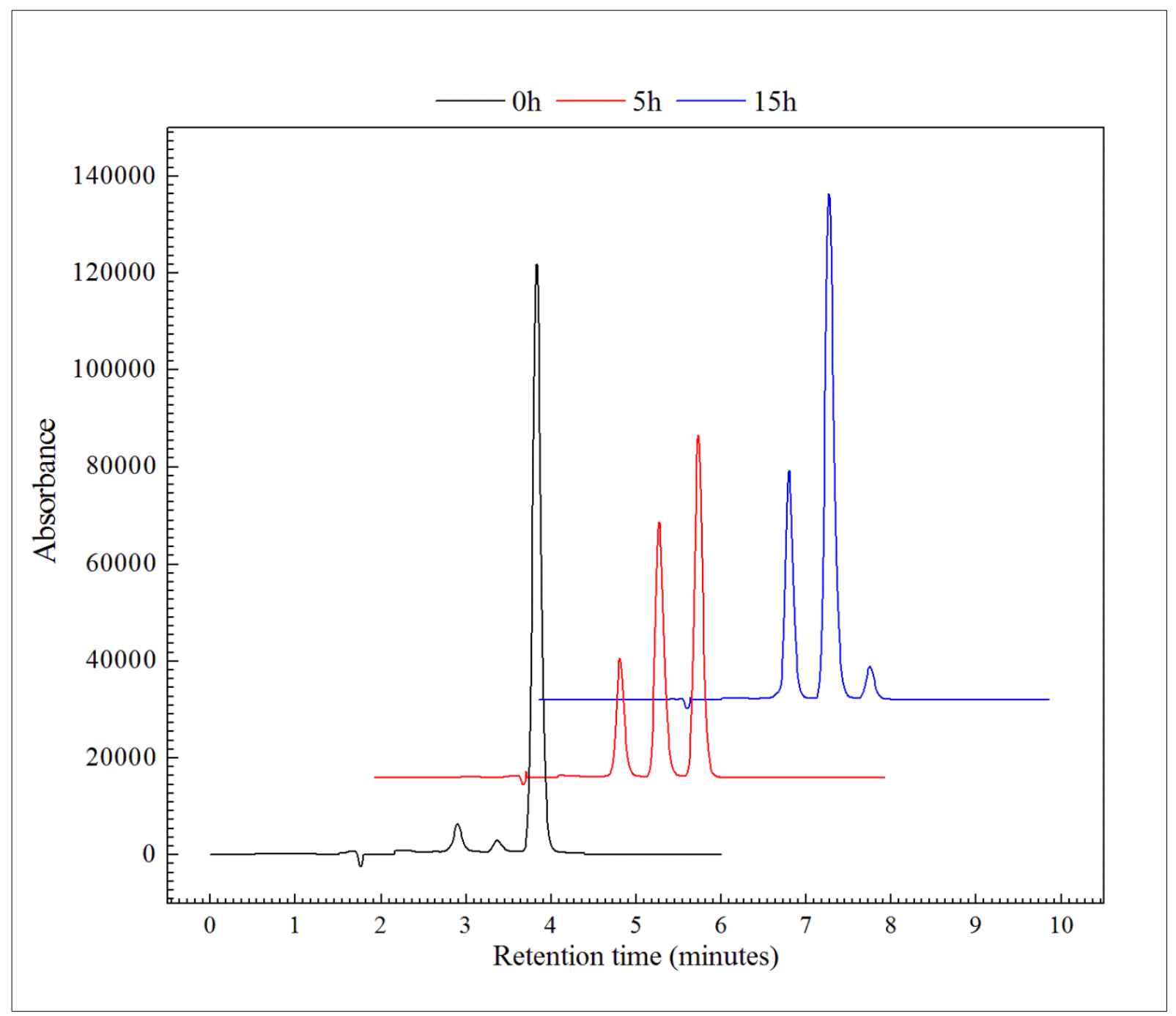

Figure 5 - Typical chromatograms resulting from stability studies of PHPGME substrate in the presence of M.La (similar profile for all PILs) including the chromatographic bands of PHPG (2.9 minutes), the unknown compound (3.4 minutes) and PHPGME (3.9 minutes). 


\section{CONCLUSION}

In order to verify the feasibility of using a promising class of solvents considered greener in the enzymatic synthesis of amoxicillin, the present study investigated two different categories of ionic liquids, protic and aprotic, as regards the stability of the substrates in the presence of these solvents. The results show that PILs are infeasible to this purpose. But, AILs seem to be very interesting and promissory to this biotechnological application, since with AILs was possible to increase the selectivity up to $400 \%$.

\section{REFERENCES}

GIORDANO, R. C.; RIBEIRO, M. P. A.; GIORDANO, R. L. C. Kinetics of $\beta$-lactam antibiotics synthesis by penicillin $G$ acylase (PGA) from the viewpoint of the industrial enzymatic reactor optimization. Biotechnol. Adv., v. 24, p. 27-41, 2006.

GREAVES, T. L.; Drummond, C. J. Protic ionic liquids: properties and applications. Chem. Rev., v. 108, p. 206-237, 2008.

OLIVIER-BOURBIGOU, H.; MAGNA, L.; MORVAN, D. Ionic liquids and catalysis: recent progress from knowledge to applications. Appl. Catal. A-Gen., v. 373, p. 1-56, 2010.

OSPINA, S. et al. Effect of $\mathrm{pH}$ in the synthesis of ampicillin by penicillin acylase. Enzyme Microb. Technol., v. 19, p. 462-469, 1996.

QUIJANO, G.; COUVERT, A.; AMRANE, A. Ionic liquids: applications and future trends in bioreactor technology. Bioresour. Technol., v. 101, p. 8923-8930, 2010.

ROOSEN, C.; MÜLLER, P; GREINER, L. Ionic liquids in biotechnology: applications and perspectives for biotransformations. Appl. Microbiol. Biotechnol., v. 81, p. 607-614, 2008.

ZHANG, Q.; ZHANG, S.; DENG, Y. Recent advances in ionic liquid catalysis. Green Chem., v. 13, p. 2619-2637, 2011.

ZHAO, H. Methods for stabilizing and activating enzymes in ionic liquids: a review. J. Chem. Technol. Biotechnol., v. 85, p. 891-907, 2010.

\section{ACKNOWLEDGEMENTS}

The authors would like to thank Brazilian research-funding agencies (CAPES, CNPq and FAPESP) and the group of the Prof. Guisán (Department of Enzymatic Biocatalysis, Institute of Catalysis, CSIC, Madrid, Spain) for the biocatalyst. 